\title{
Hybrid CNN Based Computer-Aided Diagnosis System for Choroidal Neovascularization, Diabetic Macular Edema, Drusen Disease Detection from OCT Images
}

\author{
Seda Arslan Tuncer ${ }^{*}$, Ahmet Çınar ${ }^{2}$, Murat Fırat ${ }^{3}$ \\ ${ }^{1}$ Faculty of Engineering, Software Engineering, Frrat University, Elazig 23119, Turkey \\ ${ }^{2}$ Faculty of Engineering, Computer Engineering, Firat University, Elazig 23119, Turkey \\ ${ }^{3}$ Department of Ophthalmology, Elbistan State Hospital, Kahramanmaras 46300, Elbistan, Turkey
}

Corresponding Author Email: satuncer@firat.edu.tr

https://doi.org/10.18280/ts.380314

Received: 16 November 2020

Accepted: 5 June 2021

\section{Keywords:}

choroidal neovascularization, drusen, diabetic macular edema, CNN-SVM

\begin{abstract}
In the treatment of eye diseases, optical coherence tomography (OCT) is a medical imaging method that displays biological tissue layers by taking high resolution tomographic sections at the micron level. It has an important role in the diagnosis and follow-up of many diseases such as Choroidal Neovascularization (CNV), Diabetic Macular Edema (DME), age-related macular degeneration (AMD), Diabetic Retinopathy, Central Serous Retinopathy, Epiretinal Membrane, and Macular Hole. Computer-Aided Diagnostic (CAD) tools are needed in early detection and treatment monitoring of such eye diseases. In this paper, a hybrid Convolutional Neural Networks-based CAD system, which can classify Diabetic Macular Edema (DME), Drusen Choroidal Neovascularization (CNV), and normal OCT images, is proposed. The proposed system is CNN-SVM (Convolutional Neural Networks - Support Vector Machine) model and doesn't require any additional extraction of feature or noise filtering on OCT images. A total of 968 OCT images is classified in pre-trained CNN methods with Alexnet, Resnet18 and Googlenet. Accuracy is achieved with highest Googlenet $97.4 \%$. To examine the performance of the proposed CAD system, the CNNSVM method achieves $98.96 \%$ with the highest accuracy hybrid Alexnet-SVM model, which is implemented with Alexnet-SVM, Resnet18-SVM and Googlenet-SVM models.
\end{abstract}

\section{INTRODUCTION}

Optical coherence tomography (OCT) is a medical imaging method that displays eye biological tissue layers at the micron level. Since OCT image interpretation is time-consuming, different Computer-Aided Diagnosis systems have been developed in the current years for semi / full automatic analysis of OCT images.

In the literature, there are CAD systems that use OCT in the diagnosis and classification of retinal diseases and glaucoma diseases and assist in the decision-making process of the doctor [1-3]. Zhang et al. [1] analyzed multiple ophthalmic diseases such as glaucoma, AMD, DMA using a CAD system. Rasti et al. [2] proposed a multi-scale convolutional mixture of expert (MCME) based CAD system to identify normal retina. With the CAD system developed to distinguish AMD, and DME and normal retinal images, they achieved $98.86 \%$ sensitivity and AUC 0.99985. Srinivasan et al. [3] presented a fully automated SVM-based method for the detection of retinal diseases in OCT images. SVM entries are multiscale histograms of gradient identifiers directed as feature vectors. The spectral domain OCT datasets used for cross-validation consist of volumetric scans obtained from 45 subjects. In this data set, there are 15 patients with AMD, 15 patients with DME and 15 normal images. The classifier correctly distinguished $100 \%$ of cases with AMD, 100\% with DME and $86.67 \%$ of normal subjects. Lee et al. [4] showed whether deep learning can be used to distinguish normal OCT images from images from patients with AMD. Developed to classify images as normal and AMD, the developed CAD system is a deep neural network based on VGG16. At the image level, ROC and accuracy are $87.63 \%$ and $92.78 \%$, respectively. At the macular level, OCTs are classified with $88.98 \%$ ROC and $93.83 \%$ accuracy, respectively. Liu et al. [5] proposed a machinelearning based method to identify normal macular and three types of macular pathology. The proposed system recognizes the presence of macular hole, macular edema, and AMD in the fovea-centered OCT segment. AUC value of 326 OCT images classified by SVM was obtained 0.93. Lemaitre et al. [6] examined the problem of automatically classifying Spectral Domain OCT data for automatic identification of patients with DME compared to normal people. The proposed classification consists of five different steps. The method is a combination of Local Binary Patterns (LBP) features and various mapping strategies, along with various preprocessing steps. The developed method was tested on cohort of 32 patients using linear and nonlinear classifiers. In the experimental results, normal and DME patients were classified with $81.2 \%$ sensitivity and $93.7 \%$ specificity, respectively. Das et al. [7] presented a new method for drusen (early stage) and CNV (late-stage) from DME and AMD from healthy OCT images. The method includes a multi-scale deep feature fusion-based classification approach. The MDFF fetches the inter-scale variations in images for introducing discriminative and complementary information to the classifier. The method was analyzed using 84,484 OCT images. As a result of the classification, an average of $99.6 \%, 99.87 \%$, and $99.6 \%$ sensitivity, specificity, and accuracy were obtained in the test 
set. Feng et al. [8] proposed an efficient multi-class 3D retina OCT image classification network called VinceptionC3D. VinceptionC3D is developed from basic C3D with the addition of advanced 3D inception modules. Using 873 OCT images, the proposed method was tested with three different approaches. The average accuracy achieved is $89.35 \%$, $92.09 \%$, and $94.04 \%$, respectively. Albarak et al. [9] proposed a decomposition-based approach with local feature extraction to detect the presence of AMD in OCT images. The proposed technique was applied to 140 volumetric OCT images and evaluated with tenfold cross-validation and the AUC value was obtained $94.4 \%$. Venhuizen et al. [10] designed proposed a machine learning system to automatically rate the AMD severity stages. Classified using 3265 OCT data with early, moderate, or advanced AMD symptoms, the level of AMD was analyzed using ROC analysis and Cohen's $\kappa$ statistics $(\kappa)$. The results were compared with the results determined by the two experts. AUC 0.98 , sensitivity $98.2 \%$, specificity $91.2 \%$, and $\kappa=0.713$ values were obtained. Sun et al. propose a framework for automated detection of dry AMD, and DME from OCT images. In the proposed framework, a general method is provided to automatically align and trim the retinal regions. Then, spherical representations of the images were obtained using sparse coding and a spatial pyramid. Finally, a multi-class linear SVM was preferred for classification. Two data sets were used to demonstrate the accuracy of the algorithm. For the first dataset, DME, AMD and normal subjects were classified with $100 \%, 100 \%$ and $93.33 \%$ accuracy, respectively. The second dataset was classified with $99.67 \%, 99.67 \%$ and $100 \%$ accuracy for DME, AMD and normal images, respectively Ref. [11]. Qui and Sun [12] offered self-supervised iterative refinement learning to develop a type of macular 3D OCT image classification algorithm. The presented methods were tested on a clinical data set and a general data set with 5-fold cross-validation. The proposed method provides $89.74 \%, 94.87 \%$ and $93.18 \%$ specificity, sensitivity, and accuracy, respectively, in the clinical data set. In the public data set, the results of the three metrics are $90.43 \%, 98.22 \%$, and $95.88 \%$. Perdomo et al. [13] gave the CNN-based OCT-NET architecture. The architecture is a customized CNN for processing scans from OCT volumes. OCT-NET was applied to the classification of the three states in Spectral Domain Optical Coherence Tomography-OCT volumes. In addition, the proposed model includes a feedback phase that highlights the scanning areas to support the interpretation of the results. The model has been tested on general SERI-CUHK and A2A SD-OCT datasets, including healthy, DR, DME and AMD. SERI + CUHK and A2A SDOCT datasets were used in the analysis of the proposed method, obtaining 93\% sensitivity and AUC 0.99. Fang et al. [14] developed an iterative fusion convolutional neural network for the automatic classification of OCT images. It uses an iterative fusion approach that uses features derived from convolutional layers to ensure the accurate classification of OCT images. The developed method showed better results than traditional CNN and well-known OCT classification methods. Pekala et al. detailed the latest applications of deep learning (DL) methods in the automatic fine-grained segmentation of the spectral field OCT images of the retina. Also, they have also described a new method that combines purely convolutional networks (FCN) with the Gauss Process after processing. In the proposed approach, performance comparisons between other machine learning, such as human clinicians and graphic-based approaches, were made. The approach was tested in an OCT dataset of mild nonproliferative diabetic retinopathy [15]. Acharya et al. [16] proposed a discrete wavelet transform (DWT), Radon transform (RT) and discrete cosine transform (DCT) based system for the detection of DME. In the system, sinograms were obtained from fundus images and subjected to RT. Then, DWT was applied to the sinograms to extract the wavelet coefficients. Finally, DCT was applied to obtain the 2D-DCT coefficients. In addition, coefficients are arranged in zig-zag style and converted into a 1D vector. This 1D signal was subjected to region-sensitive discriminant analysis. Finally, various controlled classifiers have been used to classify three classes using important features. The technique has provided $100 \%$ and $97.01 \%$ classification accuracy for MESSIDOR databases, using two and seven key attributes, respectively. Dufour et al. [17] proposed applying a random forest to detect the drusen positions in the retinal pigment epithelium. While the proposed method kept our computational complexity to a minimum, 0.94 AUC was achieved in the test data set. Prasath and Ramya [18] proposed a robust and adaptable algorithm for drusen detection based on Gray-Level Co-occurrence Matrixbased textural properties, reducing scan time. Localization of the Optical Disc (OD) and blood vessels using morphological operators helps to increase the accuracy of the classifier. The proposed algorithm determines the macular region based on the location of the vascular passages and OD. The proposed system provides segmentation accuracy above the ROC curve of $98.05 \%$. Yang et al. [19] reported an automated quantification algorithm design for choroidal neovascularization (CNV) in the context of neovascular AMD based on OCT Angiography images. The algorithm is based on segmentation and development methods, including Frangi filter, Gabor wavelets and Fuzzy classification. Puspitawati and Chamidah [20] classified the choroidal neovascularization using a statistical modeling approach based on a local linear estimator. According to the classification results, the accuracy is $90 \%$.

\subsection{Motivation}

In this paper, a doctorate assists CAD system for classification of Normal, Choroidal Neovascularization, DME, and Drusen OCT images is proposed. The method used is based on deep learning algorithm to categorize OCT images into CNV, Drusen, DME, and normal categories. The proposed system doesn't require any additional feature extraction or noise filtering on OCT images. SVM is used instead of Softmax layer in recommended CNN models. OCT images are given as input to $\mathrm{CNN}$ architectures and the properties in the last pooling layer of $\mathrm{CNN}$ are classified with SVM.

\subsection{Organization of paper}

The rest of the paper is organized as follows to classify OCT images with the CNN-SVM model. The data and features used in Section 2 are presented. The CAD system and AlexnetSVM structure proposed in Section 3 are detailed and the results are given. The results obtained in Section 4 are presented. In Section 5, literature comparisons are given and discussed. Finally, a brief evaluation is given in the Conclusion section. 


\section{DATA}

OCT images used in this paper were obtained from the studies $[21,22]$. In this paper, a total of 968 OCT images of 242 were evaluated for each class. Figure 1 shows four OCT images. The data set involves the well-knowing eye diseases such as CNV, Drusen, DME. Furthermore, it consists of normal image data, too.
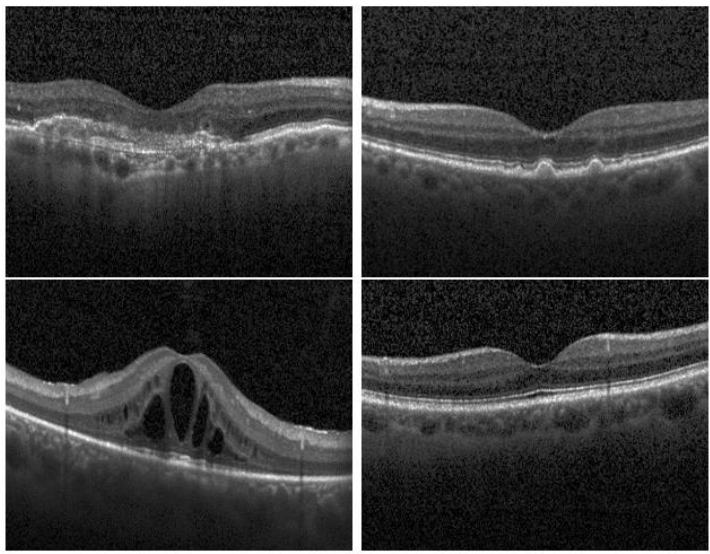

Figure 1. CNV, Drusen, DME and Normal images, respectively

\section{THE PROPOSED SYSTEM}

Computer-aided diagnosis is an interdisciplinary technology that helps doctors interpret medical images, combining artificial intelligence and computer vision. Comprehensive analysis and evaluation of images such as MRI and ultrasound in a short time is important for the expert. CAD systems process digital images to highlight prominent parts such as images and possible diseases, and support the decisions taken by the doctor. The CAD system proposed in this paper is a system that helps distinguish DME, CNV, Drusen and Normal OCT images. The formation of new blood vessels in the back of the eye and leakage in these vessels is called choroidal neovascularization. DME is also a form of vision loss for diabetic people. Extracellular substance deposits under the retinal pigment epithelium are defined as drusen. Drusen is usually studied in the context of its relationship to AMD. Some varieties of drusen are considered a normal result of aging, while others can be seen at a younger age. Also, some types of drusen are associated with different hereditary and systemic diseases. It is very important to treat such diseases in a timely manner and to prevent visual impairment and to detect early stages of CNV, DME and Drusen. For this purpose, the CAD system in Figure 2, which can classify OCT images, is proposed in the paper.

In the training phase shown in Figure 2.a, OCT images are given as input to the Alexnet-SVM model and classification accuracy is determined. In Figure 2.b the test structure of the system classifying DME, CNV, Drusen and normal OCT images can be seen. The result obtained by the system is decided by doctor control. The Alexnet-SVM architecture used in the proposed CAD system is as in Figure 3. In this proposed model, $6 \times 6 \times 256=9216$ features in the last pooling layer, where the properties of OCT images are obtained, are classified by SVM. This proposed model is a general model and other CNN models can be used instead of Alexnet.

Krizhevsky et al. [23] AlexNet, which is a convolutional neural network developed by, consists of 8 layers. The image of $227 \times 227 \times 3$ size given to AlexNet's input is reduced to $55 \times 55 \times 96$ by taking the convolution window $11 \times 11$ and Stride value 4 . This layer is followed by the max pooling layer. The data of $27 \times 27 \times 96$ size obtained in this layer is reduced to $6 \times 6 \times 25$ by applying $5 \times 5$ convolution, Max pooling and 3 times $3 \times 3$ convolution respectively. The last layer is the max pooling layer. This layer contains the properties of the OCT images given to the input. Brief definitions of layers and SVM classification algorithm used in Alexnet architecture are as follows.

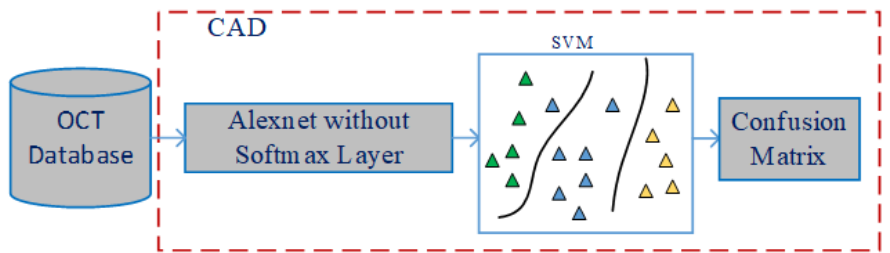

a)

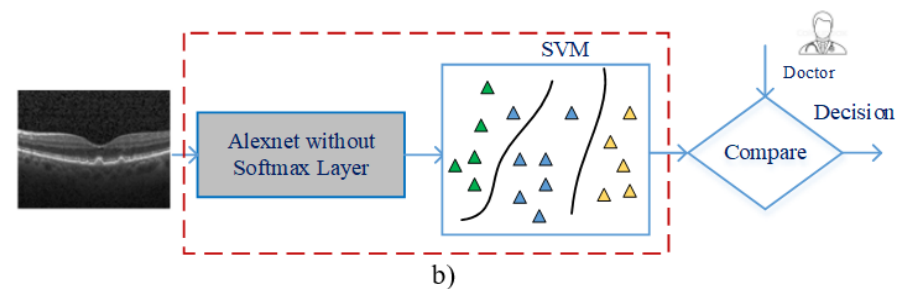

Figure 2. The proposed CAD system

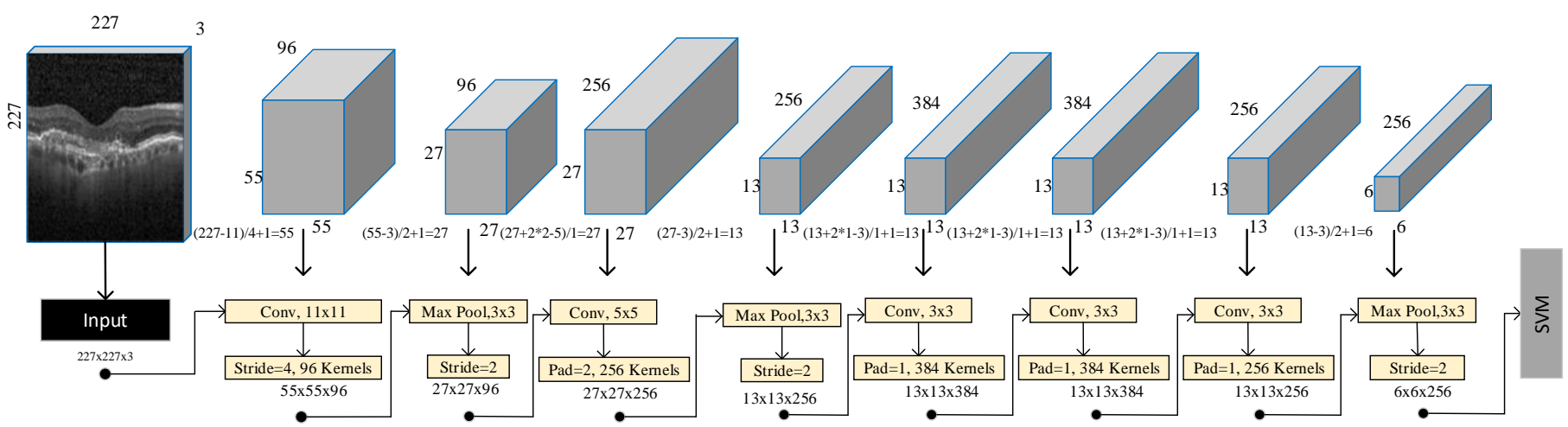

Figure 3. Architecture of Alexnet-SVM 
Convolution: This layer detects the features of the image. It applies some filters to the image to extract low and high level features in the image. Output size is calculated as in Eq. (1) with the help of input volume size (W), the receptive field size of the convolutional layer neurons $(\mathrm{F})$, applied stride $(\mathrm{S})$ and the amount of zero padding used $(\mathrm{P})$ on the border.

$$
\text { output }_{\text {size }}=\frac{W-F+2 P}{S}+1
$$

RELU: The rectified linear unit (RELU) is a nonlinear function. While the ReLU function, which is faster than functions such as Tanh, takes the value 0 for negative inputs, $\mathrm{x}$ takes the value $\mathrm{x}$ for positive inputs. It is calculated as in Eq. (2).

$$
f(x)= \begin{cases}0 & x<0 \\ x & x \geq 0\end{cases}
$$

Max Pooling: The pooling layer is usually used after a convolutional layer. The pooling layer is responsible for reducing the size of features. It aims to extract dominant features from images to speed up computation. In addition to maximum pooling, it is also used for average pooling and L2norm pooling. In practice, in the variation commonly used in the maximum pooling layer, $\mathrm{F}=3, \mathrm{~S}=2$ is used. This is also called overlapping pooling. It is calculated as in Eq. (3).

$$
\text { output }_{\text {size }}=\frac{W-F}{S}+1
$$

Normalization: Normalization layers are used between the convolution layer and the nonlinear layer to speed up network training and reduce network start sensitivity. Local Response Normalization (LRN) is used in AlexNet to help with generalization. Eq. (4) LRN gives the calculation of normalization.

$$
b_{x, y}^{i}=a_{x, y}^{i} /\left(k+\alpha \sum_{j=\max \left(0, i-\frac{n}{2}\right)}^{\min \left(N-1, i+\frac{n}{2}\right)}\left(a_{x, y}^{j}\right)^{2}\right)^{\beta}
$$

where, $\alpha$ : activation of a neuron, $\mathrm{i}$ : ith kernel, $\mathrm{N}$ : total number of kernels, $\alpha, \beta, \mathrm{k}, \mathrm{n}$ : are hyper parameters.

Support Vector Machine: SVM is one of the popular supervised learning methods used in classification problems. However, it is mostly used in classification problems. The main goal is to find the most suitable plane or hyperplane so that it can distinguish into two classes.

\section{EXPERIMENTAL RESULTS}

A total of 968 OCT images were evaluated for classification of normal, CNV, Drusen and DME OCT images. Of the 242 OCT images of each class, $70 \%$ were used for training and $30 \%$ were used for testing. Table 1 denotes training options parameters for CNN-SVM.

Confusion matrix and parameters are used to define the performance of the proposed CAD system. Table 2 describes the basic definitions of the confusion matrix. Table 3 describes the confusion matrix parameters.

In the evaluation of the classification, the Accuracy parameter is not sufficient alone, especially in unbalanced data.
Apart from this parameter, it should be evaluated in parameters such as sensitivity and fl. Sensitivity measures how often the classification gives an accurate result. Precision is a measure of how accurate it is made. While high specificity helps prevent misunderstanding (True negative), the system must be highly sensitive (True positive), especially in uncertain diagnostic situations. The F1-score is used to ignore extreme situations.

Table 1. Training option parameters

\begin{tabular}{cc}
\hline Parameter & Value \\
\hline Initial Learn Rate & 0.001 \\
Max Epoch & 15 \\
Verbose Frequency & 5 \\
Mini Batch Size & 20 \\
Optimizer & Stochastic Gradient Descent (SGDM) \\
\hline
\end{tabular}

Table 2. Confusion matrix

\begin{tabular}{cc}
\hline $\begin{array}{c}\text { True Positives } \\
\text { (TP): }\end{array}$ & $\begin{array}{c}\text { These are instances where the true value is } 1 \\
\text { and the predicted value is } 1 .\end{array}$ \\
\hline $\begin{array}{c}\text { True Negatives } \\
\text { (TN): }\end{array}$ & $\begin{array}{c}\text { These are instances where the true value is } 0 \\
\text { and our predicted value is } 0 .\end{array}$ \\
$\begin{array}{c}\text { False Positives } \\
\text { (FP): }\end{array}$ & $\begin{array}{c}\text { These are instances where the true value is } 0 \\
\text { but the predicted value is } 1 .\end{array}$ \\
$\begin{array}{c}\text { False Negatives } \\
\text { (FN): }\end{array}$ & $\begin{array}{c}\text { These are instances where the true value is } 1 \\
\text { but our predicted value is } 0 .\end{array}$ \\
\hline
\end{tabular}

Table 3. Confusion parameters

\begin{tabular}{lc}
\hline Accuracy (A) & $A=\frac{T P+T N}{T P+T N+F P+F N}$ \\
\hline Sensitivity (S) & $S=\frac{T P}{T P+F N}$ \\
Precision (P) & $P=\frac{T P}{T P+F P}$ \\
F1-Score & $F_{1}=\frac{2 T P}{2 T P+F N+F P}$ \\
\hline
\end{tabular}

Firstly, classification was carried out with the models of Alexnet, Resnet 18 and Googlenet, which are Pre-trained CNN methods. Figure 4 shows the iteration number-accuracy change and confusion matrix obtained during the training phase for the highest accuracy Googlenet. Table 4 shows the performance parameters obtained by Alexnet, Resnet18, Googlenet pre-trained CNN method.

According to the results given in Table 4, overall accuracy for Alexnet, Resnet18 and Googlenet is 96.88\%, 95.36\%, $97.40 \%$, respectively. These methods distinguish CNV, Drusen and DME disease with high accuracy in OCT images. These achievements for disease classification are at an acceptable level. However, as much as possible success is desired in the diagnosis of the disease. Therefore, in this article, hybrid models were proposed and tests were performed using SVM instead of softmax classification layers of pre-trained models. SVM classification algorithm takes the property vector of OCT images in the last pooling layer of pretrained models as input. These feature vectors are $6 \times 6 \times 256$ for Alexnet as shown in Figure 3. Feature vectors for Googlenet and Resnet 18 are $1 \times 1 \times 1024,1 \times 1 \times 512$, respectively. In the SVM algorithm, k-cros validation was applied and this value was selected as 10 . Table 5 shows the classification results obtained for Alexnet-SVM, Resnet18-SVM and GooglenetSVM. 

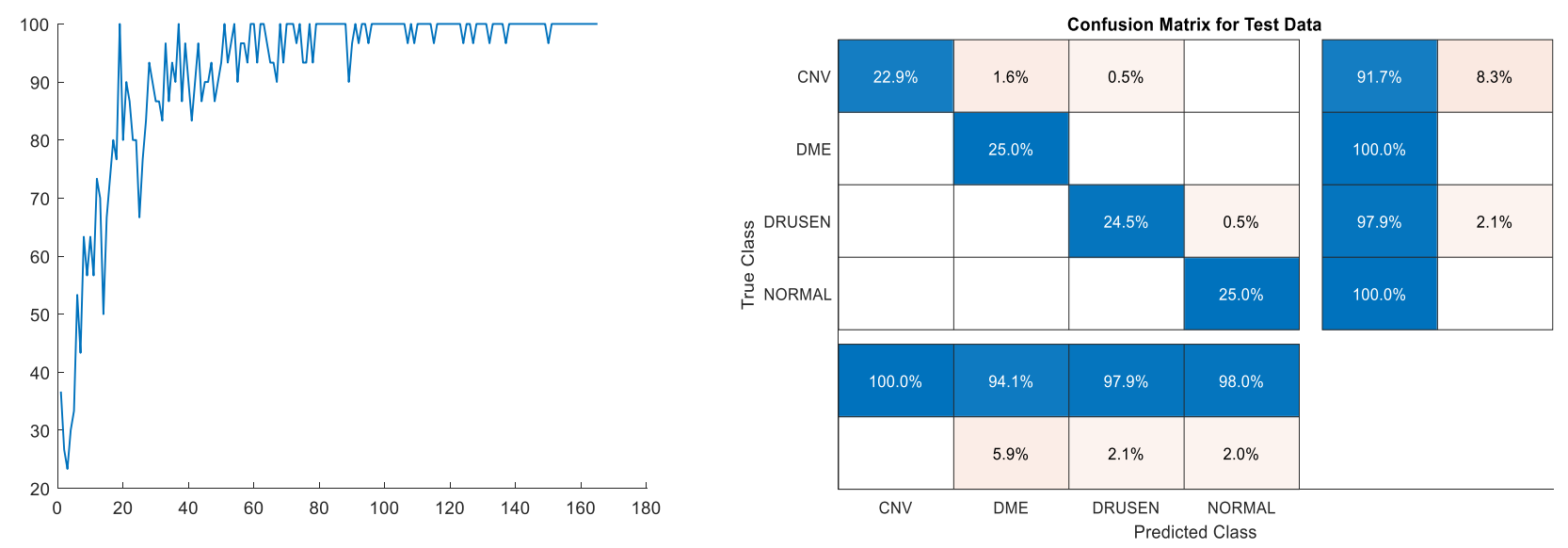

Figure 4. Googlenet accuracy change and confusion matrix

Table 4. Alexnet, Resnet18 and Googlenet performans parameters

\begin{tabular}{ccccccccccccc}
\hline Class & \multicolumn{4}{c}{ Alexnet } & \multicolumn{4}{c}{ Resnet18 } & \multicolumn{4}{c}{ Googlenet } \\
\hline & $\mathrm{A} \%$ & $\mathrm{P}$ & $\mathrm{S}$ & $\mathrm{F} 1$ & $\mathrm{~A} \%$ & $\mathrm{P}$ & $\mathrm{S}$ & $\mathrm{F} 1$ & $\mathrm{~A} \%$ & $\mathrm{P}$ & $\mathrm{S}$ & $\mathrm{F} 1$ \\
CNV & 97.4 & 0.92 & 0.98 & 0.95 & 97.68 & 0.96 & 0.95 & 0.95 & 97.92 & 0.92 & 1.0 & 0.96 \\
DME & 98.96 & 0.96 & 1.0 & 0.98 & 97.16 & 0.93 & 0.96 & 0.94 & 98.44 & 1.0 & 0.94 & 0.97 \\
Drusen & 97.92 & 1.0 & 0.92 & 0.96 & 97.68 & 0.96 & 0.95 & 0.95 & 98.96 & 0.98 & 0.98 & 0.98 \\
Normal & 99.48 & 1.0 & 0.98 & 0.99 & 98.2 & 0.97 & 0.96 & 0.96 & 99.48 & 1.0 & 0.98 & 0.99 \\
\hline
\end{tabular}

Table 5. Alexnet-SVM, Resnet18-SVM and Googlenet SVM classification results

\begin{tabular}{cccccccccccccc}
\hline Class & \multicolumn{4}{c}{ Alexnet-SVM } & \multicolumn{4}{c}{ Resnet18-SVM } & \multicolumn{4}{c}{ Googlenet-SVM } \\
\hline & $\mathrm{A} \%$ & $\mathrm{P}$ & $\mathrm{S}$ & $\mathrm{F}_{1}$ & $\mathrm{~A} \%$ & $\mathrm{P}$ & $\mathrm{S}$ & $\mathrm{F}_{1}$ & $\mathrm{~A} \%$ & $\mathrm{P}$ & $\mathrm{S}$ & $\mathrm{F}_{1}$ \\
CNV & 99.48 & 0.98 & 1.0 & 0.99 & 97.94 & 0.96 & 0.96 & 0.96 & 99.74 & 1.0 & 0.99 & 0.99 \\
DME & 99.48 & 1.0 & 0.98 & 0.99 & 97.94 & 0.95 & 0.97 & 0.96 & 100 & 1.0 & 1.0 & 1.0 \\
Drusen & 99.48 & 0.98 & 1.0 & 0.99 & 96.91 & 0.95 & 0.93 & 0.94 & 98.19 & 0.96 & 0.97 & 0.96 \\
Normal & 99.48 & 1.0 & 0.98 & 0.99 & 97.94 & 0.96 & 0.96 & 0.96 & 98.45 & 0.97 & 0.97 & 0.97 \\
\hline
\end{tabular}

Table 6. Proposed method and literature review comparison

\begin{tabular}{|c|c|c|c|}
\hline Ref. & Method & Database & Result \\
\hline Srinivasan et al. & Linear SVM, Feature Selection & 45 Cases, 3 Class (AMD, DME, Normal) & $\begin{array}{c}A=100 \% \text { for } \mathrm{AMD} \\
\mathrm{A}=100 \% \text { for } \mathrm{DME} \\
\mathrm{A}=86.67 \% \text { for Normal }\end{array}$ \\
\hline Rasti et al. & $\begin{array}{l}\text { Multi-Scale Convolution } \\
\text { Mixture of Expert }\end{array}$ & 3 Class (AMD, DME, Normal) & $\begin{array}{c}\mathrm{S}=98.86 \% \\
\mathrm{AUC}=0.9985\end{array}$ \\
\hline Lee et al. & CNN-VGG16 & 2 Class ( AMD, Normal) & $\begin{array}{c}\mathrm{A}=92.78 \text { ROC }=87.63 \% \\
\mathrm{~A}=93.83 \% \text { ROC }=88.98 \%\end{array}$ \\
\hline Liu et al. & RBF-SVM & 326 Cases, 44 Class (AMD, MH, ME, Normal) & AUC $=0.93$ \\
\hline Lemaître et.al. & $\begin{array}{l}\text { Local Binary Pattern Linear and } \\
\text { Nonlinear Classifier }\end{array}$ & 2 Class (DME, Normal) & $\begin{array}{c}\mathrm{S}=81.2 \% \\
\mathrm{Spe}=93.7 \%\end{array}$ \\
\hline Das et.al. & $\begin{array}{l}\text { CNN- Multiscale deep feature } \\
\text { fusion }\end{array}$ & 82484 Cases, 2 Class (DME, AMD) & $\begin{aligned} S & =99.6 \% \\
\text { Spe } & =99.87 \% \\
A & =99.6 \%\end{aligned}$ \\
\hline Feng et al. & VinceptionC3D & 6 Class, 873 Cases & $\mathrm{A}=94.04 \%$ \\
\hline Albarrac et al. & Bayesian Classification & 140 Cases, 2 Class(AMD, Normal) & $\mathrm{AUC}=0.944$ \\
\hline Venhuizen et al. & Random Forest Classification & 384 Cases, 2 Class( AMD, Normal) & $\begin{array}{c}\mathrm{AUC}=0.984 \\
\mathrm{CR}\end{array}$ \\
\hline Sun et al. & $\begin{array}{c}\text { SIFT Feature Extraction, Linear } \\
\text { SVM }\end{array}$ & $\begin{array}{c}\text { Set1: } 45 \text { Cases } \\
\text { Set2:678 Cases, } 3 \text { Class (AMD, DME, Normal) }\end{array}$ & $\begin{array}{c}93.93 \%, 100 \%, 100 \% \text { for Set } 1 \\
100 \%, 9967 \%, 99.67 \% \text { for Set } 2 \\
\text { Normal, AMD, DME } \\
\text { respectively }\end{array}$ \\
\hline Perdome et. al & OCT-NET & $\begin{array}{c}4 \text { Class (Normal, Diabetic Retinopathy, DME, } \\
\text { AMD) Databases: SERI-CUHK and A2A SD- } \\
\text { OCT }\end{array}$ & $\begin{array}{l}\mathrm{S}=93 \% \\
\mathrm{AUC}=0.99\end{array}$ \\
\hline $\begin{array}{l}\text { Proposed } \\
\text { Method }\end{array}$ & $\begin{array}{l}\text { CNN-SVM model, Alexnet- } \\
\text { SVM }\end{array}$ & $\begin{array}{c}968 \text { Cases, } 4 \text { Class (CNV, DME, Drusen, } \\
\text { Normal) }\end{array}$ & $\begin{array}{c}F_{1}=0.99, A=99.48 \% \text { for } C N V \\
F_{1}=0.99, A=99.48 \% \text { for } D M E \\
F_{1}=0.99, A=99.48 \% \text { for Drusen } \\
F_{1}=0.979, A=99.48 \% \text { for Normal }\end{array}$ \\
\hline
\end{tabular}



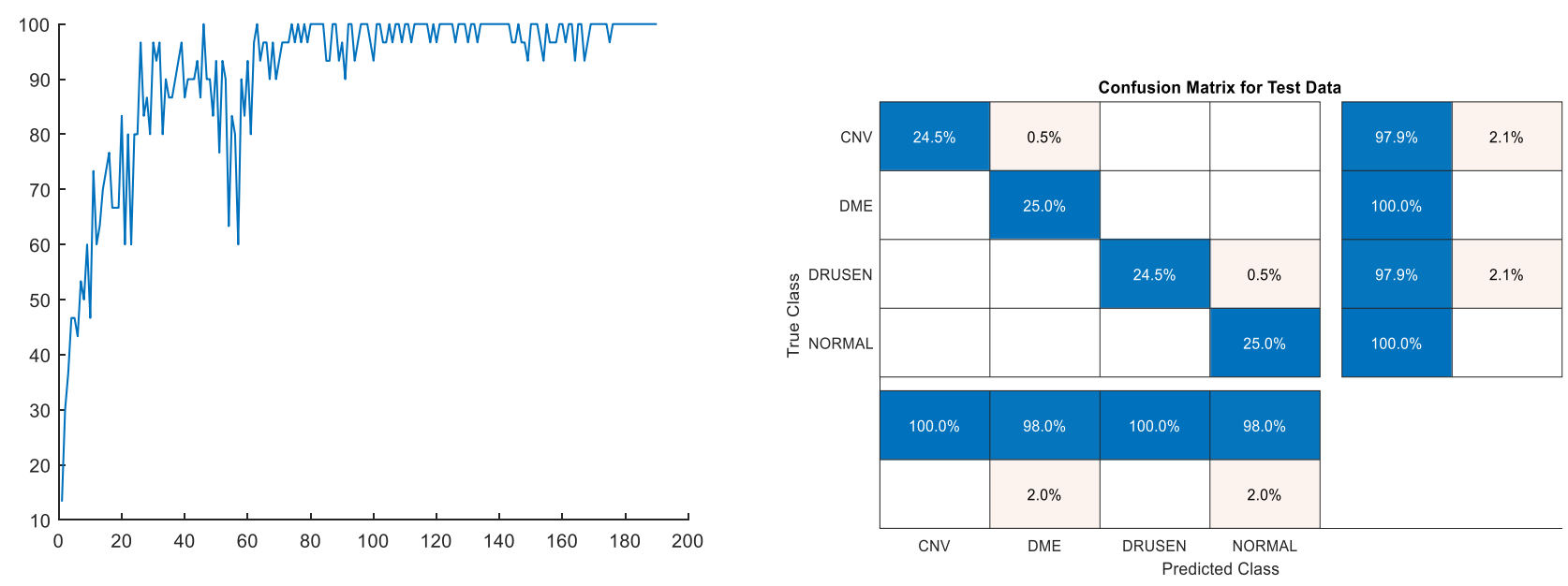

Figure 5. Alexnet-SVM accuracy change and confusion matrix

According to the results given in Table 5, overall accuracy for Alexnet-SVM, Resnet18-SVM and Googlenet-SVM is $98.96 \%, 95.36 \%, 98.20 \%$, respectively. Figure 5 shows the accuracy and confusion matrix for the Alexnet-SVM model. When all three methods are compared according to F1 parameter, more successful results have been obtained with Alexnet-SVM.

In this study, we proposed CAD based hybrid CNN method that uses SVM instead of softmax layer of pretrained CNN models to classify CNV, DME, Drusen and Normal OCT images. 968 OCT images were used to determine the accuracy of the proposed CAD system. First of all, images were classified with Alexnet, Resnet and Googlenet, which are pretrained CNN methods. The results obtained have acceptable accuracy value. To improve these results, we proposed the CNN-SVM model using the SVM classification algorithm instead of the softmax layer of pre-trained CNN models. SVM takes the display properties of the pre-trained CNN models in the pooling layer as input. The confusion matrix was used to evaluate the results and the basic classification parameters were obtained high. The comparison of the CAD system, which is a valid option for clinicians and proposed in this article, with the literature is given in Table 6.

According to Table 6, different databases, number of classes and data were used. In these studies, besides classical machine learning algorithms and statistical algorithms, it was used in CNN based algorithms. In these studies, besides accuracy, parameters such as ROC, AUC and F1 were used. Success in all these studies was over $90 \%$. It should be remembered that many criteria such as quality, number of data used, and accurate labeling of data should be taken into consideration in obtaining these results. The success of the proposed CNNSVM method has high accuracy in terms of F1 and accuracy compared to Table 5.

\section{CONCLUSION}

In this paper, a new CAD system that can classify CNV, DME, Drusen, and normal OCT images is presented and evaluated. The main contributions of this study are as follows; 1. Using as feature extractor of CNN. 2. Including of SVM model instead of Softmax layer. 3. Introducing and analyzing the CNN-SVM hybrid structures for the classification model of OCT images.
OCT images of 968, including CNV, Drusen, DME, and normal subjects, were used to demonstrate the accuracy of the proposed model. Acceptable accuracy and F1 values were obtained with Alexnet-SVM. The performance of the proposed method shows that the study can be applied in practice, diagnosis can be made accurately and quickly, and it can be suggested as a helpful system to the physician in clinical studies.

The proposed model is independent of the dataset. It can be used especially in medical image data classification, but as it is known, SVM will decrease its classification accuracy in cases where the number of classes is very high. In this case, the other classification methods can be used such as decision tree, random forest algorithm.

\section{REFERENCES}

[1] Zhang, R., He, J., Shi, S., Kang, X., Chai, W., Lu, M. (2019). Computer-aided diagnosis of ophthalmic diseases using OCT based on deep learning: A review. In International Conference on Human Centered Computing, pp. 615-625. https://doi.org/10.1007/978-3030-37429-7_63

[2] Rasti, R., Rabbani, H., Mehridehnavi, A., Hajizadeh, F. (2017). Macular OCT classification using a multi-scale convolutional neural network ensemble. IEEE Transactions on Medical Imaging, 37(4): 1024-1034. https://doi.org/10.1109/TMI.2017.2780115

[3] Srinivasan, P.P., Kim, L.A., Mettu, P.S., Cousins, S.W., Comer, G.M., Izatt, J.A., Farsiu, S. (2014). Fully automated detection of diabetic macular edema and dry age-related macular degeneration from optical coherence tomography images. Biomedical Optics Express, 5(10): 3568-3577. https://doi.org/10.1364/BOE.5.003568

[4] Lee, C.S., Baughman, D.M., Lee, A.Y. (2017). Deep learning is effective for classifying normal versus agerelated macular degeneration OCT images. Ophthalmology Retina, 1(4): 322-327. https://doi.org/10.1016/j.oret.2016.12.009

[5] Liu, Y.Y., Chen, M., Ishikawa, H., Wollstein, G., Schuman, J.S., Rehg, J.M. (2011). Automated macular pathology diagnosis in retinal OCT images using multiscale spatial pyramid and local binary patterns in texture and shape encoding. Medical Image Analysis, 15(5): 
748-759. https://doi.org/10.1016/j.media.2011.06.005

[6] Lemaître, G., Rastgoo, M., Massich, J., Cheung, C.Y., Wong, T.Y., Lamoureux, E. (2016). Classification of SD-OCT volumes using local binary patterns: Experimental validation for DME detection. Journal of Ophthalmology, 2016: 1-14. https://doi.org/10.1155/2016/3298606

[7] Das, V., Dandapat, S., Bora, P.K. (2019). Multi-scale deep feature fusion for automated classification of macular pathologies from OCT images. Biomedical Signal Processing and Control, 54: 101605. https://doi.org/10.1016/j.bspc.2019.101605

[8] Feng, S., Zhu, W., Zhao, H., Shi, F., Xiang, D., Chen, X. (2019). VinceptionC3D: A 3D convolutional neural network for retinal OCT image classification. In Medical Imaging 2019: Image Processing, 10949: 1094920. https://doi.org/10.1117/12.2509312

[9] Albarrak, A., Coenen, F., Zheng, Y. (2013). Age-related macular degeneration identification in volumetric optical coherence tomography using decomposition and local feature extraction. In Proceedings of 2013 International Conference on Medical Image, Understanding and Analysis, pp. 59-64.

[10] Venhuizen, F.G., van Ginneken, B., van Asten, F., van Grinsven, M.J., Fauser, S., Hoyng, C.B. (2017). Automated staging of age-related macular degeneration using optical coherence tomography. Investigative Ophthalmology \& Visual Science, 58(4): 2318-2328. https://doi.org/10.1167/iovs.16-20541

[11] Sun, Y., Li, S., Sun, Z. (2017). Fully automated macular pathology detection in retina optical coherence tomography images using sparse coding and dictionary learning. Journal of Biomedical Optics, 22(1): 016012. https://doi.org/10.1117/1.JBO.22.1.016012

[12] Qiu, J., Sun, Y. (2019). Self-supervised iterative refinement learning for macular OCT volumetric data classification. Computers in Biology and Medicine, 111: 103327. https://doi.org/10.1016/j.compbiomed.2019.103327

[13] Perdomo, O., Rios, H., Rodríguez, F.J., Otálora, S., Meriaudeau, F., Müller, H., González, F.A. (2019). Classification of diabetes-related retinal diseases using a deep learning approach in optical coherence tomography. Computer Methods and Programs in Biomedicine, 178: 181-189. https://doi.org/10.1016/j.cmpb.2019.06.016

[14] Fang, L., Jin, Y., Huang, L., Guo, S., Zhao, G., Chen, X. (2019). Iterative fusion convolutional neural networks for classification of optical coherence tomography images. Journal of Visual Communication and Image Representation, 59: 327-333. https://doi.org/10.1016/j.jvcir.2019.01.022

[15] Pekala, M., Joshi, N., Liu, T.A., Bressler, N.M., DeBuc, D.C., Burlina, P. (2019). Deep learning based retinal OCT segmentation. Computers in Biology and Medicine, 114:

103445 . https://doi.org/10.1016/j.compbiomed.2019.103445

[16] Acharya, U.R., Mookiah, M.R.K., Koh, J.E., Tan, J.H., Bhandary, S.V., Rao, A.K. (2017). Automated diabetic macular edema (DME) grading system using DWT, DCT features and maculopathy index. Computers in Biology and $\quad$ Medicine, 54: 59-68. https://doi.org/10.1016/j.compbiomed.2017.03.016

[17] Dufour, P.A., De Zanet, S., Wolf-Schnurrbusch, U., Kowal, J. (2012). Classification of drusen positions in optical coherence tomography data from patients with age-related macular degeneration. In Proceedings of the 21st International Conference on Pattern Recognition (ICPR2012), pp. 2067-2070.

[18] Prasath, A.R., Ramya, M.M. (2015). Detection of macular drusen based on texture descriptors. Research Journal of Information Technology, 7(1): 70-79. https://doi.org/103923/rjit.2015.70.79

[19] Yang, X., Chen, X., Xiang, D. (2020). Attention-guided channel to pixel convolution network for retinal layer segmentation with choroidal neovascularization. In Medical Imaging 2020: Image Processing, 11313: 1131334. https://doi.org/10.1117/12.2548940

[20] Puspitawati, A., Chamidah, N. (2019). Choroidal neovascularisation classification on fundus retinal images using local linear estimator. In IOP Conference Series: Materials Science and Engineering, 546(5): 052056

https://doi.org/10.1088/1757$899 \mathrm{X} / 546 / 5 / 052056$

[21] https://www.kaggle.com/paultimothymooney/kermany2 018, accessed on 19 April 2020.

[22] Kermany, D.S., Goldbaum, M., Cai, W., Valentim, C.C., Liang, H., Baxter, S.L. (2018). Identifying medical diagnoses and treatable diseases by image-based deep learning. Cell, 172(5): 1122-1131. https://doi.org/10.1016/j.cell.2018.02.010

[23] Krizhevsky, A., Sutskever, I., Hinton, G.E. (2012). Imagenet classification with deep convolutional neural networks. Advances in Neural Information Processing Systems, 25: 1097-1105. 